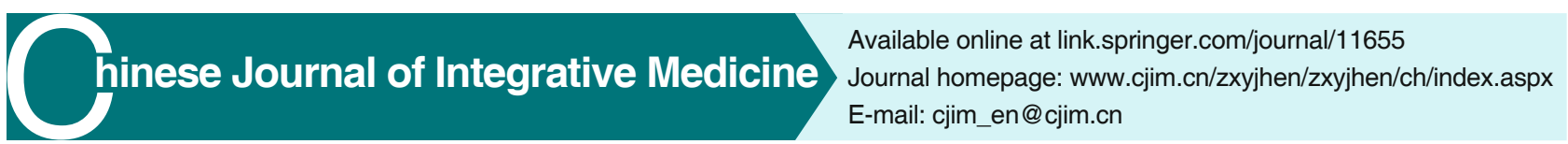

\title{
Commentary
}

\section{A Potential Chinese Medicine Granule Suppressing ARDS of COVID-19: Keguan-1}

\author{
ZHONG Nan-shan, YANG Zi-feng, JIANG Mei, and LIANG Jing-yi
}

Recently, a well-designed, exploratory clinical trial on the management of patients with coronavirus disease 2019 (COVID-19) by a Chinese medicine (CM) Keguan-1 has been conducted by Dr. XIAO Xiao-he and his colleagues. ${ }^{(1)}$ For the emergency of COVID-19 treatment, conventional new vaccine and new drug development is a money consuming and time demanding process. Consequently, it is a realistic choice to explore potential therapeutic drugs among repurposing drugs such as hydroxychloroquine and remdesivir. However, none of them lives up to the expectation for now. Under such circumstances, CM, with thousands years of medical practice including various forms of herbal medicine may be an alternative medicine approach in COVID-19 treatment. As a role model, Lianhua Qingwen Capsule (连花清瘟胶囊, LQC) - a Chinese patent medicine is proved to significantly inhibit the SARS-COV-2 replication, affects virus morphology and exerts anti-inflammatory activity in vitro. ${ }^{(2)}$ In a further clinical trial, LQC showed effectiveness and safety for the treatment of COVID-19 on the basis of usual treatment especially improving the recovery rate significantly. ${ }^{(3)}$

In despite of the fact that the pathogenesis of COVID-19 is not well revealed, it is clearly observed that progression to acute respiratory distress syndrome (ARDS) is the key turning point for the prognosis of COVID-19 patients. ${ }^{(4)}$ The death rate is closely related to ARDS and there is no approved drug for ARDS. ${ }^{(5)}$ Surprisingly, this brand new CM formula Keguan-1 was found being potential to suppress the progression to ARDS clinically. In this randomized controlled trial $(\mathrm{RCT})$, compared with the control arm, the testing arm with Keguan-1 therapy exhibited not only a significant reduction in the incidence of ARDS but also a remarkble improvement in time to fever resolution. The finding echoes with the fact that the philosophy of $\mathrm{CM}$ is characterized as holistic with emphasis on the integrity of the human body and the close relationship between human and the natural environment. $\mathrm{CM}$ focuses more on health maintenance and emphasizes on enhancing the body's resistance to diseases rather than eliminating pathogens in the treatment of disease. ${ }^{(6)}$ In face of the shortage of effective treatment, CM's unique views on life and diseases showed unprecedented advantages especially used in novel infectious disease.

This study is one of the current clinical trials to investigate the effect of CM on COVID-19, which brings us some implications. For Western medicine, it typically requires RCTs that provide statistical evidence that a drug could be successfully listed although strict pharmaceutical and preclinical studies have been completed. CM is also facing the same circumstance, high-level evidence-based clinical trials are imperatively needed to prove its efficacy with profound clinical experience in its long history of practice. First of all, sample size could be calculated in advance and to expand scale of the trial in the further study, recruiting more volunteers for validating the safety and efficacy of target medicine. Moreover, to highlight clinical value, clinical trials of $\mathrm{CM}$ are supposed to seek suitable outcome indicators combing its own advantages, such as the effect of fever resolution. Last but not least, it's also essential that statistical analysis strategies should be standardized and continuously

(C)The Chinese Journal of Integrated Traditional and Western Medicine Press and Springer-Verlag GmbH Germany, part of Springer Nature 2020

State Key Laboratory of Respiratory Disease, National Clinical Research Center for Respiratory Disease, Guangzhou Institute of Respiratory Health, the First Affiliated Hospital of Guangzhou Medical University, Guangzhou (510120), China

Correspondence to: Prof. ZHONG Nan-shan, E-mail: nanshan@ vip.163.com

DOI: https://doi.org/10.1007/s11655-020-3437-4 
optimized to ensure the authenticity and reliability of conclusions.

With the rapid development of modern biomedicine, the integration of $\mathrm{CM}$ and Western medicine to confront novel emerging infectious diseases is a new strategy and method. The clinical research team led by Dr. XIAO Xiao-he has conducted a very good exploration and practice in this area. In summary, this study set a good example for CM formula by demonstrating that $\mathrm{CM}$ with reliable quality is sufficient to provide high-quality clinical evidence for COVID-19 management and further other medical fields.

\section{REFERENCES}

1. Wang JB, Wang ZX, Jing J, Zhao P, Dong JH, Zhou $Y F$, et al. Exploring an integrative therapy for treating COVID-19: a randomized controlled trial. Chin J Integr Med 2020;26:648-655.

2. Li RF, Hou YL, Huang JC, Pan WQ, Ma QH, Shi YX, et al. Lianhuaqingwen exerts anti-viral and anti-inflammatory activity against novel coronavirus (SARS-CoV-2). Pharmacol Res 2020;156:104761.

3. Hu K, Guan WJ, Bi Y, Zhang W, Li L, Zhang B, et al. Efficacy and safety of Lianhuaqingwen Capsules, a repurposed Chinese herb, in patients with coronavirus disease 2019: a multicenter, prospective, randomized controlled trial. Phytomedicine 2020;153242. [Epub ahead of print]

4. Yang XB, Yuan Y, Xu JQ, Shu H, Xia J, Liu H, et al. Clinical course and outcomes of critically ill patients with SARSCoV-2 pneumonia in Wuhan, China: a single-centered, retrospective, observational study. Lancet Respir Med 2020;8:475-481.

5. Bauer TT, Ewig S, Rodloff AC, Müller EE. Acute respiratory distress syndrome and pneumonia: a comprehensive review of clinical data. Clin Infect Dis 2006;43:748-756.

6. Poon PMK, Wong CK, Fung KP, Fong CYS, Wong ELY, Lau JTF, et al. Immunomodulatory effects of a traditional Chinese medicine with potential antiviral activity: a selfcontrol study. Am J Chin Med 2006;34:13-21.

(Accepted August 31, 2020; First Online October 5, 2020) Edited by YUAN Lin 